taken in two planes: the first was a contact image and the second was a phase-contrast image with the detector $1.8 \mathrm{~m}$ from the object (Fig. 2a). Using the contact image and the phase-contrast image, we approximate the intensity by their average, and the intensity derivative by their difference divided by their separation. We used this approach to image a sinker made of lead aligned longitudinally with the beam (Fig. 2a, inset). The measured phase from equation (1) is shown in Fig. 2b. The phase deformity is an artefact of a gadolinium fiducial mark; the hollow core can be clearly seen in the phase image.

The phase image obtained is, to a good approximation, described by a convolution of the perfect image with the intensity distribution of the effective source. In our analysis, we used a very conservative estimate of the effective width of the neutron source to modify the phase-recovery algorithm. A profile of the recovered phase is plotted in Fig. 2c, together with the predicted phase profile determined from the sample geometry, scattering length and orientation, and gives good quantitative agreement between the two. Note that the 1,400-rad phase excursion of the sample occurs in a few hundred micrometres (less than ten pixels). An interferometric experiment would require submicrometre resolution to measure such a rapid phase excursion accurately.

This simple and general technique provides independent quantitative phase and absorption images of the sample. These images constitute two-dimensional projections of the real and imaginary parts of the neutron refractive-index distribution through a three-dimensional object. A number of these projections could be used to generate a quantitative tomographic reconstruction.

B. E. Allman ${ }^{\star}$, P. J. McMahon ${ }^{\star}$,

K. A. Nugent ${ }^{\star}$, D. Paganin ${ }^{\star}$

D. L. Jacobson $\dagger$, M. Arif $\dagger$, S. A. Werner $\ddagger$

*School of Physics, University of Melbourne,

Victoria 3010, Australia

e-mail: k.nugent@physics.unimelb.edu.au

$\dagger$ Physics Laboratory, National Institute of Standards and Technology (NIST), Gaithersburg,

Maryland 20899, USA

$\$$ Department of Physics and Astronomy,

University of Missouri-Columbia, Columbia,

Missouri 65211, USA

1. Schlenker, M. \& Baruchel, J. Physica B 137, 309-319 (1986).

2. Paganin, D. \& Nugent, K. A. Phys. Rev. Lett. 80, 2586-2589 (1998).

3. Barty, A., Nugent, K. A., Paganin, D. \& Roberts, A. Opt. Lett. 23 , 817-819 (1998).

4. Bajt, S. et al. Ultramicroscopy $83,67-73(2000)$

5. Allman, B. E. et al. J. Opt. Soc. Am. A 17, 1732-1743 (2000).

6. Nugent, K. A., Gureyev, T. E., Cookson, D., Paganin, D. \&

Barnea, Z. Phys. Rev. Lett. 77, 2961-2964 (1996).

Teague, M. R. J. Opt. Soc. Am. 73, 1434-1441 (1983).

8. Gureyev, T. E., Roberts, A. \& Nugent, K. A. J. Opt. Soc. Am. A 12, 1942-1946 (1995).

9. Dietze, M., Felber, J., Raum, K. \& Rausch, C. Nucl. Inst. Meth. A 377, 320-324 (1996).

10.Zernike, F. Physica 9, 686-693 (1942).

\section{Scaling \\ Rivers, blood and transportation networks}

- he search for a theory to explain why the metabolic rate of mammals is proportional to the 3/4-power of body mass (Kleiber's law) has recently focused on the nutrient distribution network formed by arteries and capillaries. Banavar et al. ${ }^{1}$ argue that the law follows from the intrinsic properties of an outward-directed network. But careful analysis of their arguments reveals two implicit assumptions that may not be generally correct. Unless these assumptions are valid for mammalian circulation, these arguments cannot satisfactorily explain Kleiber's empirical relationship.

In the analysis by Banavar et al. ${ }^{1}$, there is a site for nutrient uptake at each networkbranching point, and the distance $L_{X}$ along a path from the origin $O$ (the heart) to a site $X$ is defined as the number of uptake sites on the path. The rate of uptake of nutrients at a site is a constant $F_{X}$ (assumed to be speciesand size-invariant). A network segment that goes from a site $X$ to an adjacent site $Y$ is termed the link $X Y$, and the rate at which nutrient enters the link is termed the current and is denoted by $I_{X Y}$. In an outward-directed network (ODN), the direction of flow is away from $O$ on each link.

The authors' fundamental result is that, in an ODN, $C=B<L_{X}>$, where $C$, the total current in the network, is the sum of currents on all links, $B$ is the metabolic rate (sum of uptake rates), and $\left\langle L_{X}>\right.$ is the average distance to sites. The number of uptake sites is expressed as $L^{3}$, and the metabolic rate $B$ is therefore $F_{X} L^{3}$. Banavar et al. prove that, if $\left\langle L_{X}\right\rangle$ is proportional to $L$, if $C$ is proportional to blood volume $V_{\mathrm{b}}$, and if $V_{\mathrm{b}}$ is proportional to body size $M$, then $B$ is proportional to $M^{3 / 4}$, which is Kleiber's law.

Although the assumption that $V_{\mathrm{b}} \propto M$ is a reasonable approximation, the assumption that $C \propto V_{\mathrm{b}}$ is not, in general, true. Consider a network formed by $(l-1)(m-1)(n-1)$ unit cubes stacked to form a rectangle $(l-1)$ units deep by $(m-1)$ units wide by $(n-1)$ units high. Each vertex of a cube is an uptake site and each edge is a network link with constant cross-sectional area. Current is supplied to the bottom rear left corner of the network and flows forward, rightward and upward. It follows from induction on $n$ that the sum of currents in all vertical links is $\operatorname{lm} n(n+1) F_{X} / 2$. Adding the corresponding expressions for currents flowing rightward and forward gives $\operatorname{lm}_{m} F_{X}(l+m+n+3) / 2$, the total current $C$. The blood in the network is proportional to the number of links, $3 l m n-l m-l n-m n$. Thus, in this model, in which the density of uptake sites is invariant, $C$ is nearly proportional to volume multiplied by average linear dimension, metabo- lism is proportional to volume, and $V_{\mathrm{b}}$ is approximately proportional to volume.

The relation between $V_{\mathrm{b}}$ and $C$ depends on parameters that do not appear in Banavar et al.'s report ${ }^{1}$. The volume of blood in link $X Y$ is equal to the cross-sectional area of the link $\left(A_{X Y}\right)$ multiplied by the length of the link $\left(s_{X Y}\right)$, whereas current $I_{X Y}$ is $A_{X Y}$ multiplied by flow velocity $\left(f_{X Y}\right)$. The volume of blood in a link is therefore $I_{X Y} s_{X Y} / f_{X Y}$. The assumption that the sum of currents equals (or scales as) total blood volume implies that the average value of $s_{X Y}$ increases as size increases. In arteries, the flow velocity may increase as size increases, but flow rates in capillaries are severely constrained by their relatively constant crosssectional area and pressure. If most uptake sites are located in capillary networks, the currents in capillaries comprise most of the sum $C$, but the blood in these vessels may comprise a minority of blood volume.

The assumption that $\left\langle L_{X}\right\rangle \propto L$ is also not true in general in an ODN. For example, consider a network that starts with a single link and bifurcates at each branch point until a terminal uptake site is reached at distance $k$. The number of uptake sites, $L^{3}$, is $2^{k}-1$ and $\left\langle L_{X}>\right.$ is $\left[(k-1) 2^{k}-1\right] /\left(2^{k}-1\right)$. Therefore $\left\langle L_{X}\right\rangle$ is approximately proportional to the logarithm of $L$ in this type of ODN.

\section{Page R. Painter}

Office of Environmental Health Hazard Assessment, California Environmental Protection Agency,

301 Capitol Mall, Sacramento,

California 95814-4327, USA

e-mail:ppainter@oehha.ca.gov

The long-standing problem of explaining metabolic scaling ${ }^{2}$ in animals, whereby whole-animal metabolic rate $B$ is observed to increase as a function of body mass $M$ approximately as $M^{3 / 4}$, has been recently revisited by Banavar et al. ${ }^{1}$ (see also ref. 3 , in which allometric scaling rules are derived from fractal geometry). These authors ${ }^{1}$ derive and generalize to non-biological systems, including river networks, a threequarter-power 'allometric' scaling rule, which arises, in their treatment, from an assumption of the efficiency of the resource distribution network. Here I present a simple derivation of 3/4-power scaling based on the geometric requirements of inventorying resources before metabolization, which does not support the notion of allometric scaling suggested by Banavar et al. ${ }^{1}$ for rivers, at least not when applied to the problem of fluvial sediment transport. Although some distributary systems 'metabolize' according to the 3/4-power rule, this rule is not golden - each system needs to be investigated on its own merits.

As a resource distribution and processing (metabolizing) system increases in size, there is a geometric necessity for an incompressible and conserved resource for 
resource inventory in the transportation network also to increase as it awaits metabolization. Equating the rate of flow through the inventory volume to the rate of flow through the metabolizing volume provides a relation determining the scaling behaviour of the metabolization rate. Thus, if a three-dimensional metabolizing system contains two volumes, one, $V_{\mathrm{M}}$, within which metabolization of resources occurs, and one, $V_{\mathrm{I}}$, within which the resource is inventoried, and if these volumes are sequentially connected and the resource is incompressible and conserved, then $V_{\mathrm{M}} / \tau=V_{\mathrm{I}} / T$, where $\tau$ is the residence time of resource in the metabolizing volume, and $T$ is the residence time of the resource in inventory. The metabolic rate is $B \propto V_{\mathrm{M}} / \tau$.

If metabolization occurs in small volumes, $V_{\text {met }}$, of fixed size, then $V_{\mathrm{M}} \propto V_{\text {met }}(L / l)^{3}$, where $l$ is the average spacing between metabolizing volumes. The inventoried resource for each metabolizing volume $V_{\text {met }}$ is distributed in a queue that extends across the length of the system $L$, with $L / l$ units of inventory volume $V_{\mathrm{i}}$ earmarked for each volume $V_{\text {met. }}$. If $V_{\mathrm{i}}$ is independent of $L$ (which would be expected in systems where the rate-limiting process was metabolization rather than transport), then $T / \tau$ is proportional to $L / l$ (that is, the ratio of average velocities $U / u$ in the two volumes $V_{\mathrm{I}}$ and $V_{\mathrm{M}}$ is independent of $L$ ). The total inventory volume then scales as $V_{\mathrm{I}} \propto(L / l)^{4}$ (that is, $\left.l \propto L^{1 / 4}\right)$, so that $L / l \propto V_{\mathrm{I}}^{1 / 4}$, and metabolic rate is given by $B \propto V_{\mathrm{I}}^{3 / 4} / \tau$.

Three-quarter-power scaling of total metabolic rate as a function of system size follows under the additional assumptions ${ }^{1}$ that $V_{\mathrm{I}}$ ('blood' volume) is proportional to system ('whole animal') volume (or mass), and that $\tau$ is independent of system size. It is worth noting that, in the geometric argument presented here, the distribution of metabolic units, $V_{\text {met }}$, is taken to be uniform for purposes of counting, but the actual spacing can be highly non-uniform without affecting the overall scaling argument.

If allometric scaling of the kind found for animals has almost universal applicability to resource distribution systems, as suggested by Banavar et al. ${ }^{1}$, it ought to apply to rivers, an application they consider. Consider the particular case of sediment transport in rivers, in which the metabolic rate of a drainage basin of area $A$ is the rate at which sediment is delivered by the trunk stream to the lower end of the drainage basin. An analogue of blood volume $V_{\mathrm{I}}$ is the total sediment volume in transport, $V_{\text {sed }}(L / l)^{2}(T / \tau)$, where $V_{\text {sed }}$ is the volume of sediment delivered from hillslopes to stream channels from a small hillslope area $l^{2}$ during time $\tau$, and $T$ is the residence time of such sediment in the channel system of drainage basin $A$ with linear dimension $L$.

However, in fluvial systems there is no apparent basis for assuming that the velocity ratio $U / u$ is independent of $A$. It is also difficult to identify a fluvial analogue of the constraint ${ }^{1}$ that blood volume is proportional to animal volume. One difference between rivers and organisms in this regard is that, in rivers, sediment can be easily stored laterally and vertically in flood plains. Flood plains, lying outside the channels themselves, occupy a third volume representing a kind of long-term storage. Such 'loss' of sediment between hillslope and river mouth can be a significant effect ${ }^{4}$ in fluvial sediment budgets. The relation equating rates of flow in inventory and metabolizing volumes is valid only if there is no third volume for storing resources. Models of the kind proposed by Banavar et al. ${ }^{1}$ for biological organisms do not appear to be sufficient for deriving scaling relations for river (sediment) metabolism; such models potentially apply only to distribution of an incompressible resource in a system with 'two-volume' geometry.

\section{Peter K. Haff}

Division of Earth and Ocean Sciences, Nicholas School of the Environment, Duke University, Durham, North Carolina 27708-0230, USA e-mail: haff@duke.edu

Banavar et al. reply - The idea behind our theorem ${ }^{1}$ is simple. It can be illustrated by using airline travel as an example. Consider a stream of people (blood) leaving London (heart) at a steady rate and fanning out to all parts of the world (body). The number of people leaving London each day and arriving elsewhere at their final destinations (metabolic rate) is denoted by $B$. Assuming that the people travel along a locally connected network and that the transit time for each local hop is the same (say, 1 day), the number of people in transit at any given time (blood volume) is proportional to $B$, but with a proportionality constant that is given by the mean number of hops from all the destination cities to London.

This additional factor arises because if, for example, there are $P$ people who arrive in, say, Paris (let us assume Paris is two hops from London - London-Brussels-Paris) each day, there are $2 P$ passengers in transit at any given time whose final destination is Paris ( $P$ of them are en route from London to Brussels and the other $P$ are travelling from Brussels to Paris). In a $D$-dimensional space, if $B$ scales as $L^{D}$, our theorem asserts that because the mean number of hops must itself scale at least as $L$, the total number of people in transit must scale at least as $L^{D+1}$.

Haff points out the difference between the scaling properties of water flow and sediment transport in rivers and that river sediments can be stored in flood plains. Although water flow at a given point is proportional to the area of the sub-basin draining into it, the sediment discharge is not, because the source of sediments is not uniform in space, unlike the rainfall in landscape-forming events $s^{5}$. Rather, sediment production is scattered in space and time, and not isochrone with the main transport mechanisms in the network. In fluvial systems, scaling networks are stationary structures derived from the evolutionary dynamics of the topography of landscapes ${ }^{6}$.

Painter's exercise of the parallelepiped, showing that the mean number of hops from the origin, $\left\langle L_{X}\right\rangle$, is proportional to $L$ is in accord with our theorem. Painter assumes that the blood in the network is proportional to the number of links - or, by analogy, that the total number of people in transit is proportional to the number of operating flights - but this assumption is wrong because there are many more people crammed into flights originating from London than in those from cities distant from London. The number of flights is indeed proportional to the number of destinations and the metabolic rate $B$. The branching network in Painter's last paragraph is a Cayley tree which, for large enough sizes, cannot exist in any finite-dimensional space. The observation that in an $N$-site Cayley tree the average distance from the origin scales as $\ln N$, which is what would be expected for a $D$-dimensional lattice in the infinite $D$ limit, agrees with our theorem.

We do not believe that fractal-like networks effectively endow life with an additional fourth dimension ${ }^{3}$. Allometric scaling comes built in with any system in which the flow is directed and the circulation time is proportional to circulation length, irrespective of size ${ }^{1}$. The fact that nature, in spite of her extreme diversity, exhibits allometric scaling to the extent she does in plants, animals and river networks suggests that optimality associated with directedness is quite common.

Jayanth R. Banavar ${ }^{\star}$, Amos Maritan $\dagger$,

\section{Andrea Rinaldo $\ddagger$}

${ }^{*}$ Department of Physics and Center for Materials

Physics, 104 Davey Laboratory, Pennsylvania State University, University Park, Pennsylvania 16802,

USA

e-mail:banavar@psu.edu

$\dagger$ International School for Advanced Studies, Via

Beirut 2-4, 34014 Trieste, and INFM and the Abdus Salam International Center for Theoretical Physics, 34014 Trieste, Italy

$\ddagger$ Dipartimento IMAGE, Universita’ di Padova, via Loredan 20, 35131 Padova, Italy

\footnotetext{
1. Banavar, J. R., Maritan, A. \& Rinaldo, A. Nature 399, 130-132 (1999).

2. Schmidt-Nielsen, K. Scaling: Why is Animal Size so Important? (Cambridge Univ. Press, 1984).

3. West, G. B., Brown, J. H. \& Enquist, B. J. Science 284, 1677-1679 (1999).

4. Ritter, D. F., Kochel, C. R. \& Miller, J. R. Process Geomorphology 3rd edn (WCB/McGraw-Hill, Boston, 1995).

5. Rodriguez-Iturbe, I. \& Rinaldo, A. Fractal River Basins: Chance and Self-Organization (Cambridge Univ. Press, New York, 1997).

6. Banavar, J. R. et al. Phys. Rev. Lett. 78, 4522-4525 (1997).
} 